\title{
Effects of Sleep Deprivation on Weight Loss and Gut Microbiota Diversity in Obese Patients on a Calorie Restrict Diet
}

\section{Surong Wen}

Huai'an Hospital Affiliated to Xuzhou Medical University

\section{Yaojun Ni}

Hospital Affiliated to Nanjing Medical University and Huai'an First People's Hospital

\section{Ziyu Liu}

Huai'an Hospital Affiliated to Xuzhou Medical University

\section{Xiaoqing Wang}

Huai'an Hospital Affiliated to Xuzhou Medical University

\section{Jie Zhang}

Huai'an Hospital Affiliated to Xuzhou Medical University

\section{Weinan Yu}

Huai'an Hospital Affiliated to Xuzhou Medical University

Wen Hu ( $\square$ huwen787878@163.com)

Huai'an Hospital Affiliated to Xuzhou Medical University

\section{Research Article}

Keywords: Sleep deprivation, Gut microbiota, Calorie Restrict Diet, Obesity

Posted Date: December 22nd, 2021

DOI: https://doi.org/10.21203/rs.3.rs-1174529/v1

License: (c) This work is licensed under a Creative Commons Attribution 4.0 International License. Read Full License 


\section{Abstract}

Objective: This study aimed to investigate the effects of sleep deprivation (SD) on the weight loss and gut microbiota diversity in obese patients on a calorie restrict diet (CRD).

Methods: Twenty obese patients who were divided into two groups: sleep deprivation group(SD group,n=10) and non-sleep deprivation group(NSD group,$n=10$ ). All the patients received CRD for twelve weeks. Measurement of anthropometric parameters, biochemical examinations and gut microbiota detection were done at baseline and the end of week 12. MI Bands were used to monitor the sleep and exercise. Body shape parameters were measured by using the JAWON ioi353. The gut microbiota was examined by PCR in the v3-v5 region of 16S rDNA gene, and high-throughput sequencing was carried out on the lllumina Miseq platform. The operational taxonomic units (OTUs) was used for analysis.

Results: 1. CRD improved the body weight (BW), waist circumference(WC), blood pressure (BP), basal metabolic rate (BMR) ,body fat content(BFC), and Insulin resistance index (HOMA-IR) in all obese patients. 2. In the NSD group, the BW, BFC, VFA, BMR and total cholesterol (TC) reduced significantly when compared with the NSD group after CRD intervention $(P<0.05)$. 3. The Alpha diversity of gut microbiota remained unchanged after the intervention in two groups after CRD intervention. 4. There was a negative correlation between Mollicutes and BMR in the NSD group.

Conclusion: The effects of CRD may be weaken by SD in weight loss and the metabolism of blood lipid. Mollicutes bacteria may be related to the weight loss after CRD intervention in obese patients.

\section{Introduction}

Obesity has been a common disease, threatening the physical and mental health in about $39 \%$ of people worldwide ${ }^{1}$. It is also a risk factor for many diseases such as cancers, cardiovascular diseases, endocrine system diseases and digestive system diseases. Safe and effective weight loss can reduce the risk of obesity-related diseases and even decrease all-cause mortality of obese patients ${ }^{2}$. Currently, many strategies have been developed to treat obesity such as dietary intervention, exercise, drugs and surgery, and dietary intervention is the first step in the process of obesity management ${ }^{3}$. The calorie restrict diet (CRD) recommended by Chinese Nutrition Society is a nutritionally-balanced, safe and feasible strategy with favorable weight losing effect ${ }^{4}$. However, the weight losing effect varies among individuals ${ }^{5}$, but the specific mechanism is still unknown.

Sleep deprivation (SD) refers to the forced reduction of sleep duration (generally $<6 \mathrm{~h}$ within $24 \mathrm{~h}$ ) due to various reasons ${ }^{6}$. Sleep deprivation can cause a series of physiological and psychological changes, leading to a variety of adverse consequences such as cognitive decline, metabolic disorders, and finally obesity ${ }^{7}$. In addition, SD may also affect the diversity of gut microbiota, which may compromise the weight loss ${ }^{8-9}$. Therefore, this study was undertaken to investigate the effects of SD on weight loss and gut microbiota diversity in obese patients on a CRD.

\section{Materials And Methods}

\section{Subjects}

Twenty subjects with simple obesity were recruited from Huai'an Second People's Hospital between September 2018 to December 2019.

\section{Ethics statement}

This study was approved by the Ethics Committee of Huai'an Hospital, Xuzhou Medical University and Huai'an Second People's Hospital (Huai'an city, Jiangsu Province, China). 
All subjects signed the informed consent before study. This study was registered in the Clinical Trial Registration Center (No: ChiCTR1800015171).

Inclusion criteria were as follows: (1) Subjects were older than 18 years, but younger than 50 years; (2) the body mass index (BMI) was $\geq 28.0 \mathrm{~kg} / \mathrm{m}^{2}$; (3) All the subjects received the psychological assessments and CRD intervention.

Exclusion criteria were as follows: (1). Subjects were diagnosed with secondary obesity; (2) Subjects had gastrointestinal diseases or malabsorption; 3. Subjects were previously diagnosed with severe liver, kidney, heart or lung dysfunction," hypothyroidism or malignant tumor; (4) Subjects were treated with hypoglycemic drugs, lipid-lowering drugs, weight loss drugs, glucocorticoids and other drugs affecting metabolism within the last 4 weeks; (5) Subjects received weight-loss surgery before study; (6). Subjects were previously diagnosed with mental disorders, and the inability to provide self-reported data and informed consent; (7). Subjects were pregnant or breast-feeding; (8) Subjects had history of alcohol abuse (amount of alcohol consumed per week was $\geq 140 \mathrm{~g}$ for men and $\geq 70 \mathrm{~g}$ for women); (9). Subjects were previously diagnosed physical dysfunction; (10) Subjects were participated in other weight loss programs or the body weight decreased by more than $5 \%$ in the past 6 months; (11) Subjects had poorly controlled severe hypertension (systolic blood pressure $[\mathrm{SBP}] \geq 140 \mathrm{mmHg}$, diastolic blood pressure $[\mathrm{DBP}] \geq 90 \mathrm{mmHg}$ ).

Grouping: All the subjects were divided into two groups: SD group $(n=10)$ and NSD group $(n=10)$. The daily sleep time was less than $6 \mathrm{~h}$ in the SD group, but longer than $7 \mathrm{~h}$ and shorter than $9 \mathrm{~h}$ in the NSD group ${ }^{10}$. After 12-week follow-up, 2 subjects\one in SD group \anther in NSD group.》 failed to complete the intervention and 2『one in SD group \anther in NSD group. \were treated with lipid-regulating and uricase-lowering drugs or experienced a traffic accident. Thus, there were excluded from this study. Finally, sixteen subjects (NSD group, $n=8 ;$ SD group, $n=8$ ) completed this study. There were no significant differences in the gender, age, body weight (BW), body mass index (BMI) and biochemical parameters between two groups at baseline.

\section{General information collection}

After the questionnaire survey and psychological assessment, all subjects received CRD combined with appropriate exercise (at least 10,000 steps per day) for weight loss for 12 weeks.

In the CRD intervention, the daily energy intake was $20-25 \mathrm{kcal} / \mathrm{kg}$ per unit standard weight ${ }^{11}$. The standard weight $(\mathrm{kg})=$ height $(\mathrm{cm})-105$. The proportion of energy at breakfast, lunch and dinner was $30 \%, 40 \%$ and $30 \%$, respectively. The proportion of carbohydrate, protein and fat in the diet was $40 \%, 20 \%$ and $40 \%$ respectively. Meal replacement bars (Guangzhou NanDaFeiTe Nutrition Health Consulting Co. Ltd) were taken twice daily (before breakfast and before dinner). During the study, the MI Band was used to monitor the sleep and activities (the results of the MI Band met the requirements). Body shape assessment, biochemical examination, liver color ultrasonography and gut microbiota detection were performed at baseline and 12 weeks after CRD intervention.

\subsection{Questionnaire}

The demographic characteristics, lifestyle information and medical history were collected by the trained investigators through a standard questionnaire at baseline. Current smokers were defined as subjects smoking at least one cigarette per day over the past months. Drinking was defined as drinking at least once weekly for nearly 1 month.

The mental health status was assessed with the Mental Healthy Assessment Scale (Symptom Checklist 90, SCL-90) ${ }^{12}$.

\subsection{Data collection}

Body shape parameters included basal metabolic rate (BMR), body fat content (BFC), body fat percentage (BFP), visceral fat area (VFA) and BW were measured with the Korean JAWON body composition analyzer ioi353. 
The BMI was calculated as the weight $(\mathrm{kg})$ divided by the height squared $\left(\mathrm{m}^{2}\right)$. Waist circumference (WC) was measured with a non-stretchable tape over the unclothed abdomen at the narrowest point between the lowest rib and the iliac crest. Two measures were obtained and the mean (centimeters) was calculated for analyses. The blood pressure (BP) was consecutively measured thrice (OMRON Model HEM-752 FUZZY, Omron Company, Dalian, Liaoning, China), and the mean was calculated for further use.

\subsection{Blood detection}

After overnight fasting (at least $8 \mathrm{~h}$ ), blood samples were collected from the antecubital vein at 8:00 a.m 10:00 a.m. Fasting plasma glucose (FPG), total cholesterol (TC), triglycerides (TG), creatinine (Cr), urea nitrogen (BUN), uric acid (UA), fasting insulin (FIns), glutamate-pyruvate transaminase (AST) and alkaline phosphatase (ALP) were measured with standardized protocols. HbA1c was measured by high-performance liquid chromatography (Variant II and D-10 Systems, Bio-Rad Laboratories Inc, Hercules, CA, USA).

\subsection{Calculation formulas:}

Insulin resistance index $(\mathrm{HOMA-IR})=\mathrm{FPG}(\mathrm{mmol} / \mathrm{L}) \times \mathrm{FIns}(\mu \mathrm{U} / \mathrm{mL}) / 22.5^{13}$.

The estimated glomerular filtration rate (eGFR) was calculated based on the serum creatinine level by using the Chronic Kidney Disease Epidemiology Collaboration (CKD-EPI) equation ${ }^{14}$. Serum creatinine, $1 \mathrm{mg} / \mathrm{dl}=88.41 \mu \mathrm{mol} / \mathrm{l}$.

\subsection{Hepatic ultrasonography}

Hepatic ultrasonography was performed in all subjects by an experienced clinician using an Aloka Prosound a6 probe (Hitachi Aloka, Japan) with a 3-MHz probe in the same working conditions and operating standards. Before the ultrasonography, subjects experienced fasting for more than $8 \mathrm{~h}$, and lied in a left-lateral position, and ultrasonography was done during breath holding (about $5 \mathrm{~s}$ ) at the end of expiration. The mild, moderate and severe fatty liver disease was determined according to the Doppler spectrum of hepatic vein pulse and liver hemodynamics ${ }^{15}$.

\section{6 Detection of Gut microbiota}

The feces (as big as the size of soybean) (about $2 \mathrm{~g}$ ) were collected in the morning from subjects after fasting, stored separately in a sterile container at $-80^{\circ} \mathrm{C}$ at baseline and after 12-week CRD intervention. The complete genomic DNA was extracted and purified using the QIAamp DNA Stool Mini Kit (QIAGEN, Hilden, Germany) according to the manufacturer's instructions. Two-step PCR amplification was done to construct gene library. The v3-v5 region of bacterial 16S rDNA gene was amplified by PCR, high-throughput sequencing was carried out on the Illumina Miseq platform, and then the operational taxonomic unit (OTU) was analyzed and annotated with a bioinformatics software. After optimizing the gene sequence with Trimmomatic software, OTU clustering was carried out and compared with silva 123 database for species information annotation. Statistical analysis was conducted based on taxonomic information.

\section{Statistical analysis}

All statistical analyses were performed using SPSS version 22.0 (Statistical Product and Service Solutions Inc, Chicago, IL, USA). The quantitative data are expressed as means \pm standard deviations; qualitative data are expressed as numbers or percentages. Paired $t$ test was used to compare the data before and after intervention in the same group. Comparisons between groups were done using Student's t test for quantitative data and the Chi-square test for qualitative data. Stamp software was used to analyze the differences of Gut microbiota distribution. Kruskal-Wallis test was used to compare the differences in intestinal bacteria between groups, and Spearman correlation analysis was used to analyze the correlation between clinical indicators and host flora. $\mathrm{SD}_{0}$ and $\mathrm{NSD}_{0}$ represented the data at baseline in the SD group and NSD group, 
respectively. $\mathrm{SD}_{12}$ and $\mathrm{NSD}_{12}$ represented the data at the end of 12-week CRD intervention in the SD group and NSD group, respectively. A value of $\mathrm{P}<0.05$ was considered significant statistically.

\section{Results}

\section{Characteristics of all subjects before and after 12-week CRD intervention}

The WC, SBP, DBP, BFC, BW, BMR, HOMA-IR, TG and ALT,in all subjects significantly decreased after CRD intervention, as compared to those at baseline (Table 1).

Table 1. Characteristics of all subjects before and after 12-week CRD intervention.

\begin{tabular}{|c|c|c|c|}
\hline Variates & Before CRD intervention & After CRD intervention & $P$ \\
\hline$n$ & 16 & 16 & - \\
\hline $\mathrm{BMI}\left(\mathrm{kg} / \mathrm{m}^{2}\right)$ & $30.57 \pm 2.19$ & $30.52 \pm 4.45$ & 0.136 \\
\hline WC (cm) & $101.17 \pm 7.19$ & $97.37 \pm 9.06$ & 0.002 \\
\hline BW (kg) & $86.94 \pm 10.85$ & $81.54 \pm 12.02$ & 0.002 \\
\hline $\mathrm{SBP}(\mathrm{mmHg})$ & $125.56 \pm 9.93$ & $119.31 \pm 9.74$ & 0.001 \\
\hline $\mathrm{DBP}(\mathrm{mmHg})$ & $89.31 \pm 8.81$ & $82.19 \pm 5.44$ & $<0.001$ \\
\hline $\mathrm{BFC}(\mathrm{kg})$ & $28.24 \pm 4.50$ & $25.34 \pm 4.94$ & 0.020 \\
\hline BFP (\%) & $32.55 \pm 3.93$ & $30.84 \pm 4.02$ & 0.085 \\
\hline BMR (kcal) & $1489.56 \pm 187.75$ & $1451.63 \pm 187.75$ & $<0.001$ \\
\hline $\operatorname{VFA}\left(\mathrm{cm}^{2}\right)$ & $140.00 \pm 30.73$ & $123.56 \pm 32.64$ & 0.124 \\
\hline $\mathrm{HbA1c}(\%)$ & $5.46 \pm .38$ & $5.26 \pm .45$ & 0.168 \\
\hline HOMA-IR & $1.54 \pm 0.39$ & $1.35 \pm 0.34$ & 0.017 \\
\hline $\mathrm{TC}(\mathrm{mmol} / \mathrm{l})$ & $4.86 \pm 0.96$ & $4.38 \pm 0.87$ & 0.068 \\
\hline $\mathrm{TG}(\mathrm{mmol} / \mathrm{l})$ & $1.62 \pm 1.19$ & $1.07 \pm 0.77$ & 0.009 \\
\hline $\operatorname{ALT}(\mathrm{U} / \mathrm{I})$ & $35.63 \pm 26.52$ & $21.13 \pm 9.15$ & 0.028 \\
\hline AST (U/I) & $25.19 \pm 11.47$ & $19.06 \pm 4.88$ & 0.050 \\
\hline $\operatorname{ALP}(\mathrm{U} / \mathrm{I})$ & $68.75 \pm 26.01$ & $82.88 \pm 26.03$ & 0.144 \\
\hline BUN (mmol/l) & $5.57 \pm 1.03$ & $4.85 \pm 1.27$ & 0.092 \\
\hline $\operatorname{Cr}(\mu \mathrm{mol} / \mathrm{l})$ & $70.53 \pm 19.31$ & $67.59 \pm 13.60$ & 0.401 \\
\hline eGFR (ml/min) & $112.64 \pm 17.02$ & $117.03 \pm 10.1$ & 0.231 \\
\hline
\end{tabular}

Notes: All variables are expressed as n (\%) for qualitative data or mean \pm standard deviation for quantitative data.

BMI, Body mass index; WC, Waist circumference; BW, Body weight; SBP, Systolic blood pressure; DBP, Diastolic blood pressure; BFC, Body Fat Content; BFP, Body Fat Percentage; BMR, Basal metabolic rate; VFA, Visceral Fat Area; HbA1c, Hemoglobin A1C; HOMA-IR, Homeostasis model of assessment for insulin resistance index; TC, Total cholesterol; TG, Triglycerides; ALT, Alanine aminotransferase; AST, Aspartate aminotransferase; ALP, Alkaline phosphatase; BUN, Blood urea nitrogen; $\mathrm{Cr}$, creatinine; eGFR, Estimated glomerular filtration rate. 
Characteristics of subjects in two groups before and after CRD intervention

At baseline, there were no marked differences in the age, gender, BMI, WC, BW, SBP, DBP, BFC, BFP, BMR, VFA, HbA1c, HOMA-

RI, HbA1c, TC, TG, ALT, AST, ALP, BUN and eGFR between SD group and NSD group (Table 2). After CRD intervention, the BW, WC, BMR, SBP and DBP decreased significantly in both groups. However, there were slight declines in the TG in the SD group and TC,HOMA-RI in the NSD group (Table 2).

Table 2. Characteristics of subjects in two groups before and after intervention 


\begin{tabular}{|c|c|c|c|c|c|c|c|}
\hline \multirow[t]{2}{*}{ Variates } & \multicolumn{2}{|l|}{ SD } & \multirow[t]{2}{*}{$P_{a}$} & \multicolumn{2}{|l|}{ NSD } & \multirow[t]{2}{*}{$P_{b}$} & \multirow[t]{2}{*}{$P_{0}$} \\
\hline & $\begin{array}{l}\text { Before CRD } \\
\text { intervention }\end{array}$ & $\begin{array}{l}\text { After CRD } \\
\text { intervention }\end{array}$ & & $\begin{array}{l}\text { Before CRD } \\
\text { intervention }\end{array}$ & $\begin{array}{l}\text { After CRD } \\
\text { intervention }\end{array}$ & & \\
\hline$n$ & 8 & - & - & 8 & - & - & - \\
\hline $\begin{array}{l}\text { Gender } \\
\text { (Male, } \\
\%)\end{array}$ & 7 (87.5\%) & - & - & $5(62.5 \%)$ & - & - & - \\
\hline $\begin{array}{l}\text { Age } \\
\text { (years) }\end{array}$ & $38.13 \pm 2.44$ & - & - & $34.00 \pm 3.78$ & - & - & 0.160 \\
\hline $\begin{array}{l}\text { BMI } \\
\left(\mathrm{kg} / \mathrm{m}^{2}\right)\end{array}$ & $29.85 \pm 1.71$ & $29.33 \pm 1.82$ & 0.148 & $31.29 \pm 2.49$ & $29.30 \pm 6.25$ & 0.247 & 0.199 \\
\hline WC (cm) & $100.73 \pm 6.14$ & $98.60 \pm 6.76$ & 0.008 & $101.61 \pm 8.52$ & $96.14 \pm 11.26$ & 0.017 & 0.815 \\
\hline BW (kg) & $85.88 \pm 8.76$ & $83.85 \pm 9.30$ & 0.023 & $88.00 \pm 13.16$ & $79.23 \pm 14.52$ & 0.006 & 0.709 \\
\hline $\begin{array}{l}\text { SBP } \\
(\mathrm{mmHg})\end{array}$ & $128.38 \pm 7.27$ & $120.63 \pm 9.44$ & 0.019 & $122.75 \pm 11.85$ & $118.00 \pm 10.50$ & 0.012 & 0.272 \\
\hline $\begin{array}{l}\text { DBP } \\
(\mathrm{mmHg})\end{array}$ & $91.38 \pm 9.41$ & $82.88 \pm 5.84$ & 0.005 & $87.25 \pm 8.24$ & $81.50 \pm 5.32$ & 0.008 & 0.367 \\
\hline $\mathrm{BFC}(\mathrm{kg})$ & $27.11 \pm 3.28$ & $26.56 \pm 2.78$ & 0.447 & $29.36 \pm 5.44$ & $24.11 \pm 6.41$ & 0.023 & 0.334 \\
\hline BFP (\%) & $31.64 \pm 2.95$ & $31.33 \pm 2.25$ & 0.724 & $33.46 \pm 4.74$ & $30.36 \pm 5.38$ & 0.085 & 0.371 \\
\hline $\begin{array}{l}\text { BMR } \\
\text { (kcal) }\end{array}$ & $1501.75 \pm 149.36$ & $1476.25 \pm 146.63$ & 0.009 & $1477.38 \pm 229.98$ & $1427.00 \pm 228.02$ & 0.001 & 0.805 \\
\hline $\begin{array}{l}\text { VFA } \\
\left(\mathrm{cm}^{2}\right)\end{array}$ & $135.00 \pm 19.31$ & $138.25 \pm 23.34$ & 0.544 & $145.00 \pm 39.92$ & $108.88 \pm 35.29$ & 0.075 & 0.534 \\
\hline$\underset{(\%)}{\operatorname{HbA} A 1 c}$ & $5.39 \pm 0.36$ & $5.24 \pm 0.58$ & 0.540 & $5.53 \pm 0.41$ & $5.29 \pm 0.31$ & 0.168 & 0.490 \\
\hline $\begin{array}{l}\text { HOMA- } \\
\text { IR }\end{array}$ & $2.68 \pm 1.19$ & $2.15 \pm 0.84$ & 0.308 & $3.44 \pm 1.83$ & $1.91 \pm 1.78$ & 0.033 & 0.344 \\
\hline $\begin{array}{l}\text { TC } \\
(\mathrm{mmol} / \mathrm{l})\end{array}$ & $4.56 \pm 0.66$ & $4.62 \pm 0.77$ & 0.645 & $4.91 \pm 1.18$ & $4.16 \pm 0.95$ & 0.024 & 0.457 \\
\hline $\begin{array}{l}\text { TG } \\
(\mathrm{mmol} / \mathrm{l})\end{array}$ & $1.71 \pm 1.33$ & $1.20 \pm 1.01$ & 0.040 & $1.50 \pm 1.11$ & $0.94 \pm 0.45$ & 0.115 & 0.742 \\
\hline $\operatorname{ALT}(\mathrm{U} / \mathrm{I})$ & $26.75 \pm 8.46$ & $19.75 \pm 7.32$ & 0.055 & $44.50 \pm 35.43$ & $22.50 \pm 11.02$ & 0.092 & 0.206 \\
\hline $\begin{array}{l}\text { AST } \\
(\mathrm{U} / \mathrm{I})\end{array}$ & $23.38 \pm 6.80$ & $19.38 \pm 2.67$ & 0.175 & $27.00 \pm 15.10$ & $18.75 \pm 6.61$ & 0.158 & 0.546 \\
\hline $\begin{array}{l}\text { ALP } \\
(U / I)\end{array}$ & $60.75 \pm 20.53$ & $72.88 \pm 27.2$ & 0.418 & $76.75 \pm 29.68$ & $92.87 \pm 21.94$ & 0.242 & 0.230 \\
\hline $\begin{array}{l}\text { BUN } \\
(\mathrm{mmol} / \mathrm{l})\end{array}$ & $5.28 \pm 0.94$ & $4.77 \pm 1.05$ & 0.457 & $5.85 \pm 1.09$ & $4.93 \pm 1.52$ & 0.107 & 0.277 \\
\hline $\begin{array}{l}\mathrm{Cr} \\
(\mu \mathrm{mol} / \mathrm{l})\end{array}$ & $73.12 \pm 18.67$ & $72.11 \pm 11.95$ & 0.855 & $67.94 \pm 20.86$ & $63.08 \pm 14.37$ & 0.309 & 0.609 \\
\hline $\begin{array}{l}\text { eGFR } \\
\text { (ml/min) }\end{array}$ & $110.97 \pm 16.36$ & $113.42 \pm 10.61$ & 0.654 & $114.32 \pm 18.62$ & $120.65 \pm 8.79$ & 0.243 & 0.708 \\
\hline
\end{tabular}


Notes: All variables are expressed as $\mathrm{n}(\%)$ for qualitative data or mean \pm standard deviation for quantitative data. Pa, the difference of the change of each parameter before and after intervention in the SD group. $\mathrm{Pb}$, the difference of the change of each parameter before and after intervention in the NSD group. P0, the difference of baseline parameters between SD group and NSD group. NSD, non-sleep deprivation group. SD, sleep deprivation group. BMI, Body mass index; WCm Waist circumference; BWm Body weight; SBP, Systolic blood pressure; DBP, Diastolic blood pressure; BFC, Body Fat Content; BFP, Body Fat Percentage; BMR, Basal metabolic rate; VFA, Visceral Fat Area; HbA1c, Hemoglobin A1C; HOMA-IR, Homeostasis model of assessment for insulin resistance index; TC, Total cholesterol; TG, Triglycerides; ALT, Alanine aminotransferase; AST, Aspartate aminotransferase; ALP, Alkaline phosphatase; BUN, Blood urea nitrogen; Cr, creatinine; eGFR, Estimated glomerular filtration rate

\section{Changes in the parameters in two groups before and after the CRD intervention}

After intervention, the mean BFC, VFA, BW, BMR and TC in the NSD group decreased by $4.70 \mathrm{~kg}, 39.38 \mathrm{~cm}^{2}, 6.74 \mathrm{~kg}, 24.88$ $\mathrm{kcal}$ and $0.83 \mathrm{mmol} / \mathrm{l}$, respectively, and these changes were more obvious than those in the SD group. However, there were no significant differences in the changes of BMI, BFP, WC, ALT, AST, ALP, BUN, eGFR, TG, HOMA-RI,HbA1c, SBP and DBP between NSD group and SD group (Table 3).

Table 3. Changes in the body parameters and biochemical parameters after CRD intervention in two groups.

\begin{tabular}{|llll|}
\hline Variates & $\mathrm{SD}$ & $\mathrm{NSD}$ & $\boldsymbol{P}$ \\
\hline $\boldsymbol{n}$ & 8 & 8 & - \\
\hline$\Delta \mathrm{BMI}\left(\mathrm{kg} / \mathrm{m}^{2}\right)$ & $0.53 \pm 0.91$ & $1.99 \pm 4.45$ & 0.378 \\
\hline$\Delta \mathrm{WC}(\mathrm{CM})$ & $2.13 \pm 1.63$ & $5.48 \pm 4.99$ & 0.107 \\
\hline$\Delta \mathrm{BW}(\mathrm{kg})$ & $2.02 \pm 1.97$ & $8.76 \pm 6.36$ & 0.013 \\
\hline$\Delta \mathrm{SBP}(\mathrm{mmHg})$ & $7.75 \pm 7.25$ & $4.75 \pm 3.99$ & 0.327 \\
\hline$\Delta \mathrm{DBP}(\mathrm{mmHg})$ & $8.50 \pm 6.00$ & $5.75 \pm 4.40$ & 0.314 \\
\hline$\Delta \mathrm{BFC}(\mathrm{kg})$ & $0.55 \pm 1.93$ & $5.25 \pm 5.10$ & 0.029 \\
\hline$\Delta \mathrm{BFP}(\%)$ & $0.31 \pm 2.40$ & $3.10 \pm 4.38$ & 0.137 \\
\hline$\Delta \mathrm{BMR}(\mathrm{kcal})$ & $25.50 \pm 20.04$ & $50.38 \pm 23.77$ & 0.040 \\
\hline$\left.\Delta \mathrm{VFA}(\mathrm{cm})^{2}\right)$ & $-3.25 \pm 14.41$ & $36.13 \pm 48.94$ & 0.047 \\
\hline$\Delta \mathrm{HbA} 1 \mathrm{c}$ & $0.15 \pm 0.66$ & $0.23 \pm 0.42$ & 0.179 \\
\hline$\Delta \mathrm{HOMA}-\mathrm{RI}$ & $0.53 \pm 1.36$ & $1.53 \pm 1.64$ & 0.904 \\
\hline$\Delta \mathrm{TC}(\mathrm{mmol} / \mathrm{l})$ & $-0.06 \pm 0.37$ & $0.77 \pm 0.76$ & 0.014 \\
\hline$\Delta \mathrm{TG}(\mathrm{mmol} / \mathrm{l})$ & $0.051 \pm 0.57$ & $0.56 \pm 0.881$ & 0.895 \\
\hline$\Delta \mathrm{ALT}(\mathrm{U} / \mathrm{l})$ & $7.00 \pm 8.59$ & $22.00 \pm 31.85$ & 0.219 \\
\hline$\Delta \mathrm{AST}(\mathrm{U} / \mathrm{l})$ & $4.00 \pm 7.50$ & $8.25 \pm 14.75$ & 0.480 \\
\hline$\Delta \mathrm{ALP}(\mathrm{U} / \mathrm{l})$ & $-12.13 \pm 39.87$ & $-16.13 \pm 35.69$ & 0.836 \\
\hline$\Delta \mathrm{BUN}(\mathrm{mmol} / \mathrm{l})$ & $0.51 \pm 1.82$ & $0.92 \pm 1.41$ & 0.615 \\
\hline$\Delta \mathrm{Cr}(\mu \mathrm{mol} / \mathrm{l})$ & $1.01 \pm 15.17$ & $4.86 \pm 12.55$ & 0.589 \\
\hline$\Delta \mathrm{eGFR}(\mathrm{ml} / \mathrm{min})$ & $2.45 \pm 14.78$ & $6.33 \pm 14.03$ & 0.599 \\
\hline
\end{tabular}


Note: All variables are expressed as $\mathrm{n}(\%)$ for qualitative data or mean \pm standard deviation for quantitative data. NSD, nonsleep deprivation group. SD, sleep deprivation group. BMI, Body mass index; WC, Waist circumference; BW, Body weight; SBP, Systolic blood pressure; DBP, Diastolic blood pressure; BFC, Body Fat Content; BFP, Body Fat Percentage; BMR, Basal metabolic rate; VFA, Visceral Fat Area; HbA1c, Hemoglobin A1C; HOMA-IR, Homeostasis model of assessment for insulin resistance index; TC, Total cholesterol; TG, Triglycerides; ALT, Alanine aminotransferase; AST, Aspartate aminotransferase; ALP ,Alkaline phosphatase; BUN, Blood urea nitrogen; $\mathrm{Cr}$, creatinine; eGFR, Estimated glomerular filtration rate. " $\Delta$ ": difference between baseline level and 12-week level.

\section{Findings from Doppler ultrasonography}

At baseline, fatty liver disease was noted in all the subjects of both SD group and NSD group. After 12-week intervention, fatty liver disease was not observed in $87.5 \%$ and $37.5 \%$ of subjects in the NSD group and SD group, respectively.

\section{Gut microbiota distribution}

Sequencing depth of gut microbiota: the species accumulation curve of samples was analyzed by random sampling method to detect the sequencing depth. The results showed that the dilution curve tended to flatten when there were 30 sequencing samples, indicating that the depth of this sequencing met the requirements, and the additional amount of sequencing data was not necessary for studying the discovery of OTU (Figure 1).

\section{Gut microbiota in two groups before and after CRD intervention}

As shown in Fig.2A, the numbers of OTUs were similar between $\mathrm{SD}_{0}$ and $\mathrm{SD}_{12}, \mathrm{NSD}_{0}$ and $\mathrm{NSD}_{12}, \mathrm{SD}_{0}$ and $\mathrm{NSD}_{0}$, and between $\mathrm{SD}_{12}$ and $\mathrm{NSD}_{12}\left(\mathrm{P}_{\mathrm{SD} 0-\mathrm{SD} 12}=0.57, \mathrm{P}_{\mathrm{NSD} 0-\mathrm{NSD} 12}=0.08, \mathrm{P}_{\mathrm{SD} 0-\mathrm{NSD0}}=0.29\right.$ and $\left.\mathrm{P}_{\mathrm{SD} 12-\mathrm{NSD12}}=0.43\right)$. However, as shown in Fig.2B, the abundance in the NSD group increased gradually after intervention, while that in the SD group decreased gradually, and the abundance in the SD group was lower than in the NSD group before and after CRD intervention,without significance.

\section{Heat map analysis of correlation between gut microbiota abundance and clinical factors before and after intervention in two groups}

At the phylum level, heat maps were plotted based on the correlation coefficient between abundance of gut microbiota and clinical indicators. As shown in Figure 3, at baseline, in the NSD group, Selenomonadales was negatively related to AST, Bifidobacteriaceae and $\mathrm{Cr}$. Coriobacteriaceae were negatively related to FBG. Desulfovibrionaceae was positively related to BFR. There was a negative correlation between Mollicutes and BMR.

However, there was no significant association in the SD group between abundance of gut microbiota and clinical factors.

At the end of 12 weeks after CRD intervention (Fig.4), in the NSD group, there was a significant positive correlation between Akkermansia and FBG. Bifidobacteriaceae was positively related to FBG, HbA1C and TG. Clostridiales was negatively related to BUN. There was a negative correlation between Mollicutes and BMR. However, in the SD group, Burkholderiales was positively related to FBG. Desulfovibrionaceae was positively related to AU. Actinobacteria and Actinomycetaceae were negatively related to VFA.

\section{Mollicutes Alpha diversity in two groups before and after intervention.}

A stable negative correlation between Mollicutes bacteria and BMR was noted in the NSD group before and after CRD intervention (Fig3-4). To better analyze the relations between Mollicutes and BMR, Mollicutes Alpha diversity was analyzed separately in the Figure 5. There were no significant difference in the relative abundance of Mollicutes before and after intervention in the SD group and NSD group $\left(P_{S D 0-S D 12}=0.685, P_{N S D 0-N S D 12}=0.524\right)$, However, the change of relative abundance of Mollicutes in the NSD group after intervention was significantly greater than in the SD group. 


\section{Discussion}

It is estimated that more than $44 \%$ of the global population have overweight, and more than 300 million people are affected by obesity. In the past decade, the incidence of obesity has increased at an alarming rate ${ }^{16}$. Obesity has been a risk factor for many diseases, including type 2 diabetes mellitus (T2DM), nonalcoholic fatty liver disease and cardiovascular disease ${ }^{17}$. Therefore, obesity has been a global public health problem that should be managed actively. Our study showed, after 12 weeks of CRD and exercise intervention, some clinical parameters including WC, BW, SBP, DBP, BFC, BMR, HOMA-IR, TG and ALT significantly decreased. It suggests that CRD intervention can effectively reduce the BW and BFC, improve fat metabolism and relieve insulin resistance even in the presence of SD. Although this has been reported previously 18-19, findings in previous studies were from obese patients with T2DM, and there were no prospective studies. In our study, all subjects were diagnosed with simple obesity and CRD program was designed by a professional nutritionist and sports manager before study. Therefore, our results may be helpful to improve the management of obesity.

Studies have indicated that SD may promote the occurrence and development of obesity in children and adults ${ }^{20-21}$, however the relationship between SD and weight loss is poorly understood. Our study showed that CRD intervention had a good effect on the weight loss in all subjects and improved WC, BW, BMR,SBP and DBP. When the subjects were divided into SD and NSD group, results showed the BFC and TC in the NSD group significantly reduced after intervention, while both remained unchanged in the SD group. In addition, the changes of VFA, BW, BFC, BMR and TC in the NSD group were more obvious as compared to those in the SD group after CRD intervention. This further indicates that SD compromises the protective effect of CRD on the weight loss and lipid metabolism. Moreover, the prevalence of fatty liver disease in the NSD group and SD group reduced after weight loss following 12-week intervention, 87.5\% of subjects in the NSD group had no fatty liver disease, which was significantly higher than in the SD group (37.5\%). Therefore, SD may also compromise the improvement of fatty liver disease during the CRD intervention. However, the specific mechanism is unclear. There are several possible explanations, (1) SD may lead to uncontrollable behaviors and poor diet compliance in the process of weight loss 20,22 ; (2) sleep deprivation may decrease leptin, increase ghrelin as well as hunger and appetite, which all cause a decrease in the basal metabolic rate and an increase in the intake, ultimately leading to obesity ${ }^{23}$; (3) SD may damage the compositions of gut microbiota ${ }^{24}$, thereby increasing intestinal permeability, compromising the metabolism of nutrients such as fat and sugar and finally resulting in low energy consumption and ultimately obesity ${ }^{25}$. Among these mechanisms, the most remarkable one is that SD damages the diversity of gut microbiota and thus compromises the protective effects of CRD.

To further investigate the potential mechanism, the gut microbiota was detected before and after CRD intervention in all subjects. 32 samples were examined before and after intervention, and the dilution curve results showed, if the number of samples was greater than 30 , the measured data volume was reasonable. Thus, the number of samples in this study met the requirements. Studies have reported that diet can quickly and effectively change the compositions of gut microbiota ${ }^{26-}$ 27. However, no significant differences were found in the alpha diversity analysis of intergroup differences in gut microbiota (Fig.2A). However, the abundance of the curve of gut microbiota at baseline and the end of 12 weeks after intervention changed to a certain extent. Results showed that, after 12-week intervention, the abundance of gut microbiota in the NSD group was the highest, and that in the SD group was almost no change. Another study also shows that SD had a certain effect on the abundance of gut microbiota ${ }^{28}$, which may affect the regulatory effect of diet on the gut microbiota. The reason why the gut microbiota abundance of the NSD group after CRD intervention was higher than that of the SD group after intervention might be ascribed to that SD may gradually compromise the rapid regulatory effect of diet on the gut microbiota.

In addition, the relationships between gut microbiota and clinical factors were evaluated in all the subjects. There were close correlations between species abundance of gut microbiota and clinical factors, and the diversity of gut microbiota in the SD group was inhibited at baseline and had no relationship with clinical factors. Most of these correlations were unstable and

Page 10/18 
special, but a stable negative correlation between Mollicutes bacteria and BMR was noted in the NSD group before and after CRD intervention. Compared with the SD group, the relative abundance of Mollicutes increased significantly in the NSD group. However, there was no similar stable correlation between BMR and abundance of Mollicutes in the SD group. Therefore, we speculate that SD disturbs the dynamic balance of gut microbiota,which leads to the stable correlation between BMR and abundance of Mollicutes to be broken. A study has shown that the recovery of low BMR is a sign of better weight loss ${ }^{29}$. Therefore, in obese patients without SD, increasing the abundance of Mollicutes may be beneficial to reduce BMR and improve the weight loss effect of CRD. However, the specific mechanism should be elucidated in future studies.

Our study showed that CRD intervention reduced SBP and DBP in obese subjects with or without SD, but the change of DBP in the NSD group was more obvious than that in the SD group. A Study suggests that SD compromises the antihypertensive effect of CRD due to the SD induced increase of $B P{ }^{30}$. Interestingly, the change of SBP in the SD group was more obvious than in the NSD group. This might be ascribed to the higher SBP at baseline in the SD group and thus the BP was sensitive to dietary intervention, but this should be further confirmed in more studies.

\section{Conclusions}

In conclusion, our results indicate that CRD intervention may effectively reduce the BW, WC, BMR, SBP and DBP of obese subjects and also improve lipid metabolism in these subjects with normal sleep rhythms. SD may impair the weight loss effects of CRD intervention and inhibit the diversity of gut microbiota in simple obese subjects. Our study for the first time investigates the relationships among SD, CRD intervention and gut microbiota. Our findings suggest that Mollicutes bacteria may be an important factor affecting the weight loss effect of diet intervention in obese patients with SD. A further study on the Mollicutes may provide an important basis for the treatment of obesity by targeting gut microbiota in the future. Unfortunately, there were limitations in our study,(1) this was a single center, prospective clinical study with small sample size. (2) the CRD intervention was administered for only 12 weeks, which was relatively short. Therefore, more studies with large sample size are needed to confirm our findings and investigate the effects of long term CRD intervention in obese subjects, which may provide a new strategy for the clinical management of obesity.

\section{Abbreviations}

sleep deprivation $\mathbb{S D}$

calorie restrict diet $\triangle \mathrm{CRD}$

non-sleep deprivation group $₫ \mathrm{NSD}$

operational taxonomic units $\mathbb{O} \mathrm{OTUS}$

body weight $₫ \mathrm{BW}$

waist circumference $₫ \mathrm{WC}$

blood pressure $₫ \mathrm{BP}$

basal metabolic rate $\mathbb{B} \mathrm{BR}$

body fat content $\triangle \mathrm{BFC}$

Insulin resistance index $\triangle \mathrm{HOMA}-\mathrm{IR}$

total cholesterol $\mathbb{T C}$ 


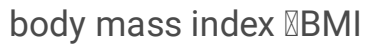

(systolic blood pressure $\mathbb{}$ SBP

diastolic blood pressure $\triangle \mathrm{DBP}$

body mass indexखBMI

body fat content $\triangle \mathrm{BFC}$

body fat percentage $\triangle B F P$

visceral fat area $\triangle \mathrm{VFA}$

Waist circumference $₫ \mathrm{WC}$

Fasting plasma glucose $\triangle \mathrm{FPG}$

total cholesterol $₫ \mathrm{TC}$

triglycerides $\triangle T \mathrm{TG}$

creatinine $₫ \mathrm{Cr}$

urea nitrogen $\triangle \mathrm{BUN}$

uric acid $₫ \mathrm{UA}$

fasting insulin $\mathbb{F}$ Ins

glutamate-pyruvate transaminase $₫ A S T$

alkaline phosphatase $\triangle A L P$

Insulin resistance index $\triangle \mathrm{HOMA}-\mathrm{IR}$

estimated glomerular filtration rate囚eGFR

\section{Declarations}

\section{Authorship}

All named authors meet the International Committee of Medical Journal Editors (ICMJE) criteria for authorship for this article, take responsibility for the integrity of the work as a whole, and have given their approval for this version to be published.

\section{Disclosures}

WeiNan Yu, SuRong Wen, YaoJun Ni, XiaoQing Wang, ZiYu Liu, Jie Zhang and Wen Hu have nothing to declare.

\section{Ethics approval and consent to participate}

This study was approved by the Ethics Committee of the Second Huai'an People's Hospital, and informed consent was obtained from each subject or their family members before study. All procedures performed in studies involving human 
participants were in accordance with the ethical standards of the institutional research committee and with the 1964 Helsinki Declaration and its later amendments or comparable ethical standards.

\section{Consent for publication}

Consent to publish has been obtained from the participant to report individual patient data.

\section{Availability of data and materials}

The datasets during and/or analysed during the current study available from the corresponding author on reasonable request.

\section{Competing interests}

The authors declare that they have no competing interests.

\section{Funding}

This study was supported by the National Natural Science Foundation of China (81700776) and Health Research Project of Huai'an City, Jiangsu (HAWJ201911).

\section{Authors' contributions}

Wen Hu, SuRong Wen and WeiNan Yu are responsible for the conception and design of the article, analyzing and interpreting the results, writing papers and paper revisions; Wen Hu and SuRong Wen conducted feasibility analysis, participating in the writing of papers and paper revisions; SuRong Wen, YaoJun Ni, ZiYu Liu and Xiaoqing Wang participated in the implementation of the study and data collection; SuRong Wen and Jie Zhang conducted data collation and statistical processing; Wen Hu is responsible for the quality control, review and supervision of the article and is responsible for the overall article.

\section{Acknowledgements}

The authors thank all participants for their cooperation and sample contribution.

\section{Authors' information}

Surong Wen and Yaojun Ni are co-first authors and contributed equally to this work. Wen Hu and Weinan Yu are Correspondingauthors and contributed to this paper equally.

\section{References}

1. Levesque RJR. Obesity and Overweight: Springer New York: 2017.

2. Zheng W, Mclerran DF, Rolland B, Zhang X, Potter JD. Association between Body-Mass Index and Risk of Death in More Than 1 Million Asians. N Engl J Med 2011; 364(8): 719-29.

3. Lisbeth MV, James T, Michael F, et al. World Gastroenterology Organisation global guidelines on obesity. J CLIN GASTROENTEROL 2012; 46(7): 555-61.

4. China CCCO. Consensus of medical nutrition therapy experts for overweight/obesity in China (2016 edition). Chinese Journal of Diabetes Mellitus 2016; 8(10): 525-40.

5. Cox L, Blaser M. Pathways in Microbe-Induced Obesity. CELL METAB 2013; 17(6): 883-94.

6. Sanches I, Teixeira F, Santos JMD, Ferreira AJ. Effects of Acute Sleep Deprivation Resulting from Night Shift Work on Young Doctors. Acta Med Port 2015; 28(4). 
7. Reynolds AC, Paterson JL, Ferguson SA, Stanley D, Dawson D. The shift work and health research agenda: Considering changes in gut microbiota as a pathway linking shift work, sleep loss and circadian misalignment, and metabolic disease. SLEEP MED REV 2016; 34.

8. Ming C, Huiwen X, Dan L, et al. Circadian Rhythm Shapes the Gut Microbiota Affecting Host Radiosensitivity. INT J MOL SCI 2016; 17(11): 1786.

9. Ley RE, Turnbaugh PJ, Samuel K, Gordon Jl. Microbial ecology: human gut microbes associated with obesity. NATURE 2006; 444(7122): 1022-3.

10. Hirshkowitz M, Whiton K, Albert SM, et al. National Sleep Foundation's updated sleep duration recommendations: final report. Sleep Health 2015: S1140205050.

11. Toscani MK, Mario FM, Radavelli-Bagatini S, Wiltgen D, Matos MC, Spritzer PM. Effect of high-protein or normal-protein diet on weight loss, body composition, hormone, and metabolic profile in southern Brazilian women with polycystic ovary syndrome: a randomized study. Gynecological Endocrinology the Official Journal of the International Society of Gynecological Endocrinology 2011; 27(11): 6.

12. Hoz EV. Symptom Check-List-90-R1975.

13. Matthews D. Homeostasis model assessment : insulin resistance and beta-cell function from fasting plasma glucose and insulin concentrations in man. DIABETOLOGIA 1985; 28(7): 412.

14. Rao MK. Chronic Kidney Disease: National Clinical Guideline for Early Identification and Management in Adults in Primary and Secondary Care. KIDNEY INT 2009; 75(6): 571.

15. BaoQi Li HXJW. Application value of color doppler ultrasound in the detection of hemodynamic changes in patients with nonalcoholic fatty liver disease. Chinese General Practice 2013; 2(16): 130-2.

16. $\mathrm{Ng} \mathrm{T}$. Global, regional, and national prevalence of overweight and obesity in children and adults during 1980-2003: a systematic analysis for the Global Burden of Disease Study 20132014; 384(9945): 766-81.

17. Després JP, Lemieux I. Abdominal obesity and metabolic syndrome. NATURE 2006; 444(7121): 881-7.

18. GBD Obesity Collaborators, MH AAF, Reitsma MB, et al. Health Effects of Overweight and Obesity in 195 Countries over 25 Years. NEW ENGL J MED 2017.

19. Zhao L, Zhang F, Ding X, Wu G, Zhang C. Gut bacteria selectively promoted by dietary fibers alleviate type 2 diabetes. SCIENCE 2018; 359(6380): 1151-6.

20. Chaput J, Dutil C. Lack of sleep as a contributor to obesity in adolescents: impacts on eating and activity behaviors. INT J BEHAV NUTR PHY 2016; 13(1): 103.

21. Mchill AW, Wright KP. Role of sleep and circadian disruption on energy expenditure and in metabolic predisposition to human obesity and metabolic disease. Obesity Reviews An Official Journal of the International Association for the Study of Obesity 2017; 18: 15-24.

22. Chen SM, Liu CY, Li SR, Huang HT, Jou HJ. Effects of Therapeutic Lifestyle Program on Ultrasound-diagnosed Nonalcoholic Fatty Liver Disease. J CHIN MED ASSOC 2008; 71(11): 551-8.

23. Turnbaugh PJ, Ridaura VK, Faith JJ, Rey FE, Knight R, Gordon JI. The Effect of Diet on the Human Gut Microbiome: A Metagenomic Analysis in Humanized Gnotobiotic Mice2009; 1(6): 6.

24. Wang Y, Kuang Z, Yu X, Ruhn KA, Kubo M, Hooper LV. The intestinal microbiota regulates body composition through NFIL3 and the circadian clock. SCIENCE 2017; 357.

25. Thaiss CA, Zeevi D, Levy M, Zilberman-Schapira G, Suez J. Transkingdom Control of Microbiota Diurnal Oscillations Promotes Metabolic Homeostasis. CELL 2014; 159(3): 514-29.

26. David LA, Maurice CF, Carmody RN, et al. Diet rapidly and reproducibly alters the human gut microbiome. NATURE 2014; 505(7484): 559.

27. Bowyer RCE, Jackson MA, Pallister T, et al. Use of dietary indices to control for diet in human gut microbiota studies. MICROBIOME 2018; 6(1): 77.

Page 14/18 
28. Steinberg DM, Christy J, Batch BC, et al. Preventing Weight Gain Improves Sleep Quality Among Black Women: Results from a RCT. Annals of Behavioral Medicine A Publication of the Society of Behavioral Medicine 2017.

29. Soares MJ, Cummings NK, Ping-Delfos WLCS. Energy metabolism and the metabolic syndrome: Does a lower basal metabolic rate signal recovery following weight loss? Diabetes Metab Syndr 2011; 5(2): 98-101.

30. Riegel B, Daus M, Lozano AJ, Malone SK, Patterson F, Hanlon AL. Shift Workers Have Higher Blood Pressure Medicine Use, But Only When They Are Short Sleepers: A Longitudinal UK Biobank Study. J AM HEART ASSOC 2019; 8(20).

\section{Image}

Please see the Supplemental Files section for the doppler ultrasound images of fatty and normal liver.

\section{Figures}

\section{Species Accumulation Curves}

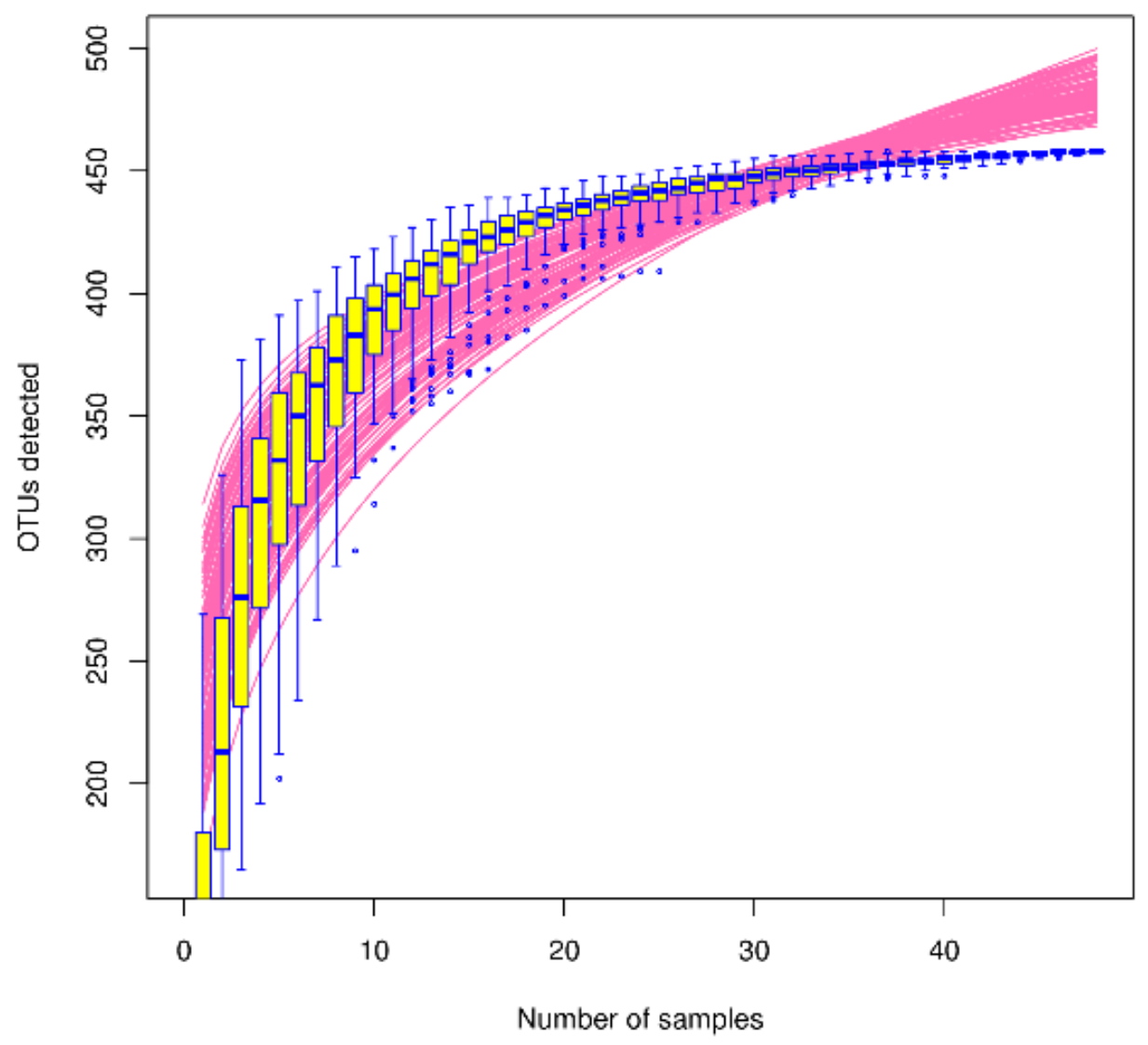

Figure 1

Species accumulation curve of sequencing depth test 
Fig 2A

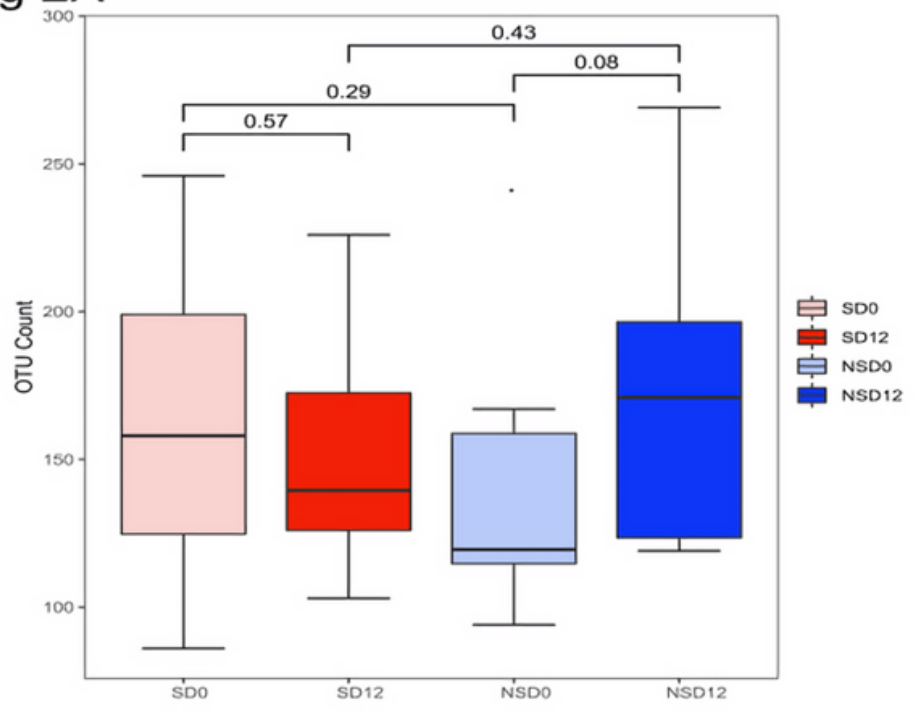

Fig 2B Rank-abundance distribution Curves

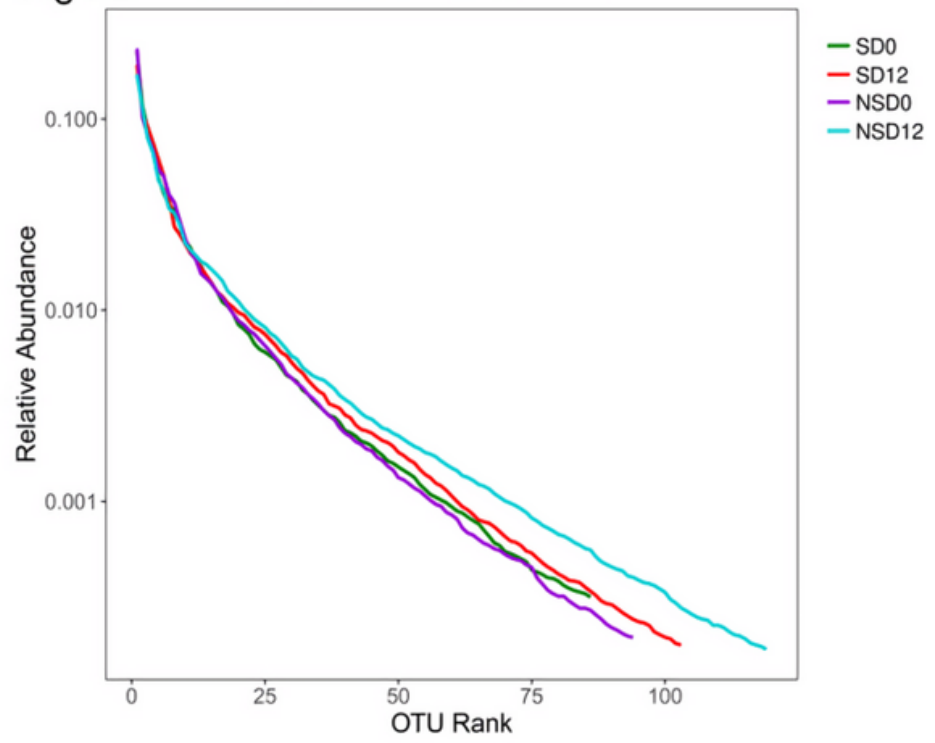

Figure 2

A. Gut microbiota abundance Alpha diversity between SD group and NSD group before and after intervention. B. Curve analysis of gut microbiota and species Rank-abundance.

Note: $\mathrm{SD}_{0}$ : baseline in the SD group, $\mathrm{SD}_{12}$ : the end of 12 weeks after CRD intervention in the SD group, $\mathrm{NSD}_{0}:$ baseline in NSD group, $\mathrm{NSD}_{12}$ : the end of 12 weeks after CRD intervention in the NSD group.

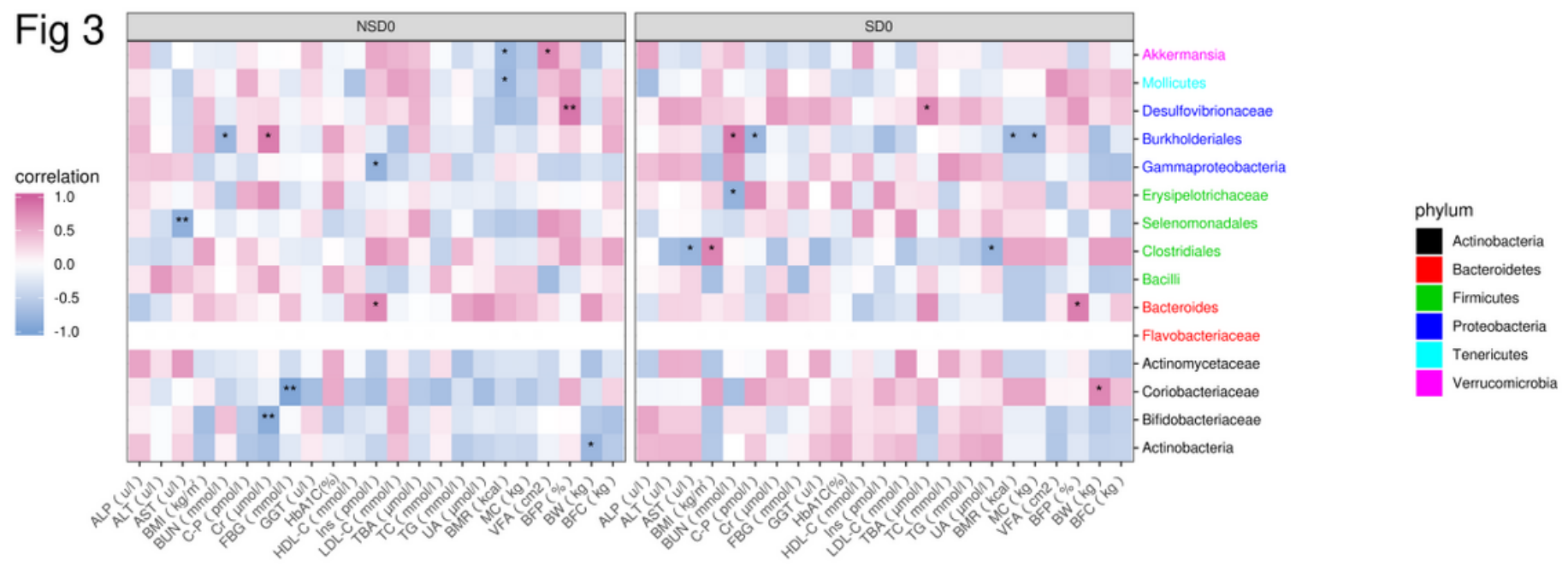

Figure 3

Heat map analysis of correlation between gut microbiota of phylum and clinical indicators at baseline

Note: MC Muscle Content, C-P C-peptide, Cr Creatinine, FBG Fasting plasma glucose, GGT y-glutamyl transpeptidase, HDL-C High density lipoprptein cholesterol, LDL-C low density lipoprptein cholesterol, TBA Total bile acid, UA Uric acid, BMI Body mass index, BFC Body Fat Content, BFP Body Fat Percentage, VFA Visceral Fat Area, BW Body weight, WC Waist circumference, BMR Basal metabolic rate, ALT, Alanine aminotransferase, AST, Aspartate aminotransferase, ALP, Alkaline 
phosphatase, BUN, Blood urea nitrogen, TC, Total cholesterol, TG Triglycerides, HbA1c, hemoglobin A1C,SD 0 baseline in the sleep deprivation group, $\mathrm{NSD}_{0}$ baseline in non-sleep deprivation group, ${ }^{*} \mathrm{P}<0.05 \rrbracket \star \star \mathrm{P}<0.01$.

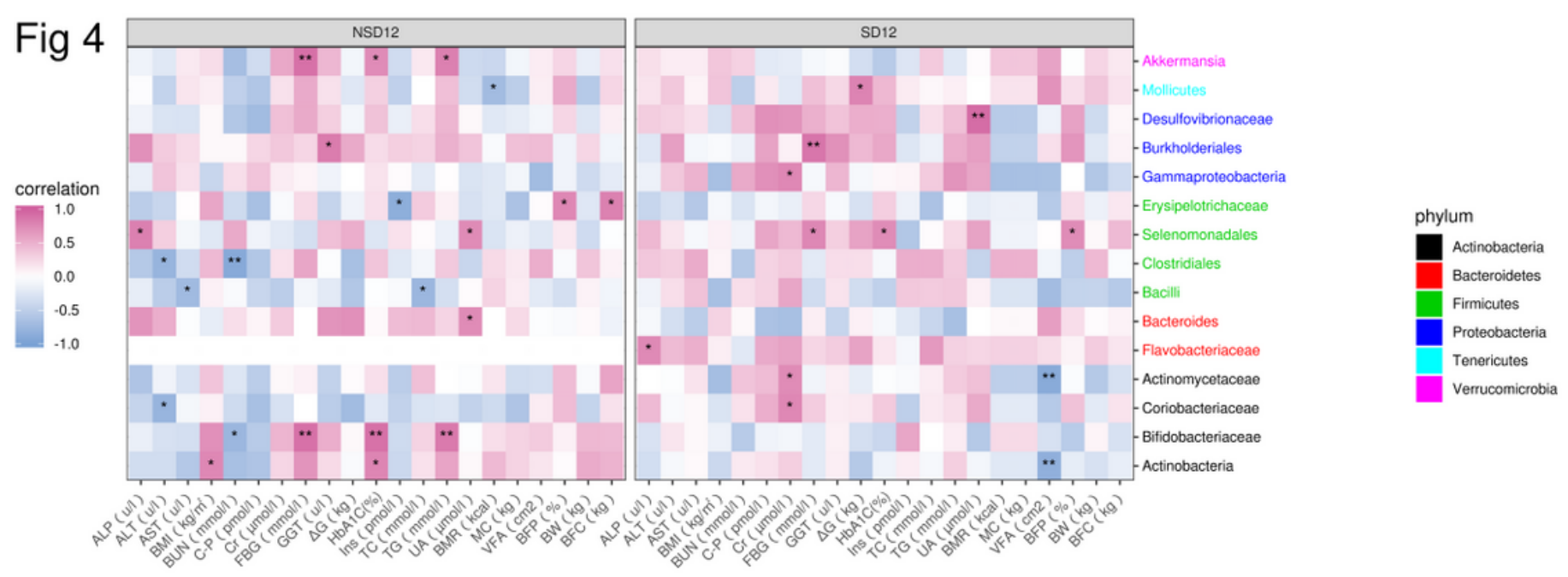

Figure 4

\section{Heat map analysis of correlation between gut microbiota at the level of phylum intervention and clinical indicators}

Note: MC Muscle Content, C-P C-peptide, Cr Creatinine, FBG Fasting plasma glucose, GGT Y-glutamyl transpeptidase, HDL-C High density lipoprptein cholesterol, LDL-C low density lipoprptein cholesterol, TBA Total bile acid, UA Uric acid, BMI Body mass index, BFC Body Fat Content, BFP Body Fat Percentage, VFA Visceral Fat Area, BW Body weight, WC Waist circumference, BMR Basal metabolic rate, ALT, Alanine aminotransferase, AST, Aspartate aminotransferase, ALP, Alkaline phosphatase, BUN, Blood urea nitrogen, TC, Total cholesterol, TG Triglycerides, HbA1c, hemoglobin A1C, SD12: the end of 12 weeks after CRD intervention in the sleep deprivation group, NSD12: the end of 12 weeks after CRD intervention in the nonsleep deprivation group. * $p<0.05 \rrbracket^{* *} p<0.01$. 


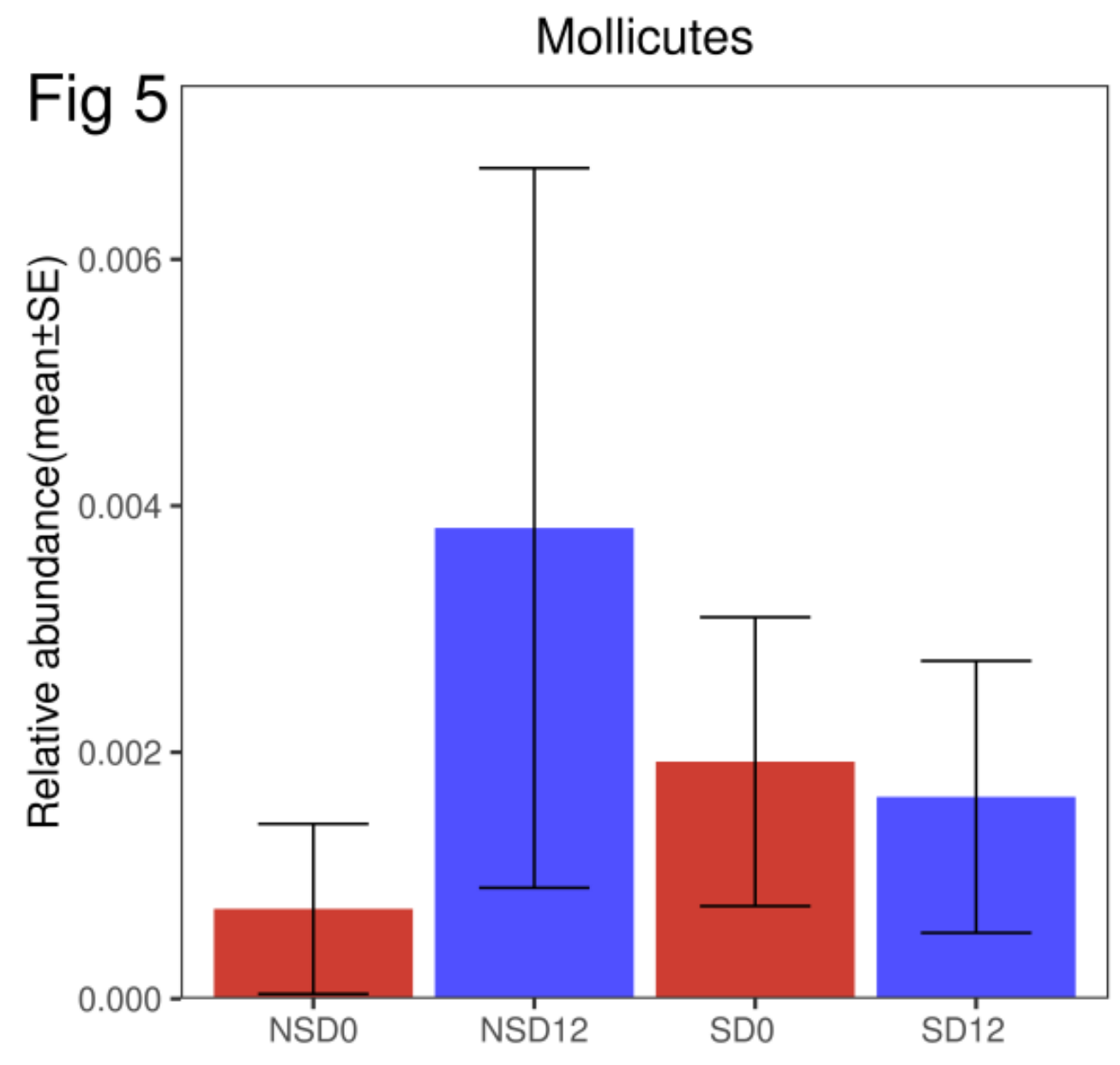

Figure 5

Mollicutes Alpha diversity in two groups before and after intervention.

Note: $\mathrm{P}_{\text {SD0-SD12 }}$, the change of Mollicutes in the SD group after 12-week intervention. PNSD0-NSD12, the change of Mollicutes in the NSD group after 12-week intervention. SD0: baseline in the SD group, NSD0: baseline in NSD group, SD12: the end of 12 weeks after CRD intervention in the SD group, NSD12: the end of 12 weeks after CRD intervention in the NSD group. ${ }^{*} \mathrm{P}<0.05$.

\section{Supplementary Files}

This is a list of supplementary files associated with this preprint. Click to download.

- 1.png 